\title{
Guide de publication de la recherche scientifique en sciences de la santé
}

\author{
P Huston ${ }^{1,2}$, BCK Choi $^{2-4 *}$
}

\section{Résumé}

Une communication efficace de la recherche scientifique est essentielle pour faire progresser la science et pour optimiser l'influence de son propre travail professionnel. Cet article fournit un guide sur la préparation des articles scientifiques qui vont être publiés en sciences de la santé. Il s'adresse aux professionnels de la santé qui commencent à communiquer leurs résultats dans des revues examinées par les pairs ou qui aimeraient rafraîchir leurs connaissances dans ce domaine. Il définit cinq étapes clés. Premièrement, il faut adopter des pratiques exemplaires en matière de publications scientifiques, y compris la rédaction collaborative et l'éthique dans la production de rapports. Deuxièmement, il faut positionner votre article de manière stratégique avant d'entamer la rédaction. II suffit de définir votre public cible, de choisir trois à cinq revues qui touchent ce public cible, puis de vous informer au sujet des exigences des revues. Troisièmement, il faut que vous créiez la première ébauche de votre article en préparant un scénario logique, concis et convaincant en fonction des exigences relatives aux revues et de la structure établie pour les articles scientifiques. Quatrièmement, il faut peaufiner l'article en coordonnant la contribution de vos coauteurs et en appliquant des principes de bonne composition et de rédaction claire. La version définitive de l'article doit répondre aux exigences rédactionnelles et être approuvée par tous les auteurs avant la présentation. Cinquièmement, une fois l'article soumis, il faut se préparer à la révision. Les rejets sont courants; si vous recevez des commentaires, il faut que vous envisagiez de réviser le document avant de l'envoyer à une autre revue. Si la revue accepte votre article, il faut tenir compte de toutes les révisions demandées. Les articles scientifiques qui ont un impact important ne sont pas seulement un bon fondement scientifique; ils sont également très lisibles et sont le fruit d'un effort collectif et souvent synergique.

\author{
Affiliations \\ 1 Direction générale de la \\ prévention et du contrôle des \\ maladies infectieuses, Agence \\ de la santé publique du Canada, \\ Ottawa (Ontario) \\ 2 École d'épidémiologie et \\ de santé publique, Université \\ d'Ottawa, Ottawa (Ontario) \\ ${ }^{3}$ Direction générale de la \\ promotion de la santé et de \\ la prévention des maladies \\ chroniques, Agence de la santé \\ publique du Canada, Ottawa \\ (Ontario) \\ ${ }^{4}$ Injury Prevention Research \\ Center, Shantou University \\ Medical College, Shantou (Chine)
}

*Correspondance : bernard. choi@phac-aspc.gc.ca

Citation proposée : Huston P, Choi BCK. Guide de publication de la recherche scientifique en sciences de la santé. Relevé des maladies transmissibles au Canada. 2017;43(9):191-8.

https://doi.org/10.14745/ccdr.v43i09a01f

\section{Introduction}

La publication des résultats de la recherche scientifique est importante pour deux raisons. D'abord, la progression de la science dépend de la publication des résultats de recherche dans des documents évalués par des pairs. Ensuite, la publication de la recherche est importante pour le perfectionnement professionnel. Le vieux dicton "publier ou périr " évoque le rôle crucial joué par la recherche scientifique, surtout pour les personnes du milieu universitaire. La version plus récente, "publier et prospérer ", indique que la publication de recherches scientifiques rigoureuses est bonne pour chaque chercheur et pour la communauté scientifique. Avec de bonnes recherches, chacun serait mieux loti.

La publication de travaux scientifiques n'est pas chose aisée. Il existe de nombreux livres sur la façon de rédiger un article scientifique (1-5); toutefois, le degré de détail peut être écrasant et on a tendance à se concentrer davantage sur les aspects techniques, notamment la structure d'un article scientifique et les éléments à inclure dans chaque section, et moins sur les aspects du processus, particulièrement les éléments qui constituent la paternité et la manière de choisir la revue la plus appropriée. Un survol de base est nécessaire pour les personnes qui souhaitent commencer à publier ou à rafraîchir leurs connaissances dans ce domaine. Cet article vise à donner aux professionnels de la santé un aperçu de la manière de préparer des articles destinés à la publication.

\section{Adoption de pratiques exemplaires dans les publications scientifiques}

Quiconque souhaite écrire des publications scientifiques devrait connaître ces deux pratiques exemplaires avant de commencer : le travail en collaboration et le respect de pratiques éthiques dans la production de rapports.

\section{Pratique de la rédaction collaborative}

La recherche et les publications scientifiques sont des entreprises collectives qui appellent à la collaboration en tant que pratique exemplaire. Habituellement, la recherche réunit une équipe de recherche. Les nouveaux projets de recherche s'appuient 
sur les recherches précédentes réalisées par d'autres. Cela suppose la contribution des pairs à la fois dans l'élaboration des protocoles avant la réalisation des recherches et dans l'examen des articles une fois les recherches terminées. La Collaboration Cochrane en est un important exemple (6). Afin d'optimiser la réussite de votre équipe de recherche, vous devez cultiver de fortes aptitudes interpersonnelles et choisir vos collaborateurs avec soin. Les domaines à prendre en considération au moment de choisir avec qui travailler comprennent notamment les éléments tels que la disponibilité des collaborateurs, les intérêts semblables en matière de recherche, la réputation, ainsi que les qualités personnelles.

Étant donné qu'une publication scientifique vise à contribuer aux connaissances, une bonne question de recherche est essentielle, de même que la détermination de la méthode scientifique optimale pour répondre à cette question et le respect de pratiques éthiques dans la réalisation de vos recherches. Une fois ces points traités, que devez-vous savoir avant d'entamer la rédaction?

\section{Respect de pratiques éthiques dans la production de rapports}

L'éthique des publications scientifiques peut se résumer en deux pratiques exemplaires : la production de rapports complets et exacts et la reconnaissance pertinente des contributions de chacun (7).

\section{Assurer la production de rapports complets et exacts}

Par pratiques de publication scientifique contraires à l'éthique, on entend notamment la production de rapports incomplets, la publication des données frauduleuses, le plagiat, les publications faisant double emploi, et les publications se chevauchant. Certaines personnes considèrent que l'omission de publier les résultats d'essais cliniques est contraire à l'éthique (8), car cela peut créer un biais dans le dossier publié. La production de rapports incomplète peut comprendre la déclaration sélective de résultats ou carrément l'absence de communication de ces résultats. Il est important de communiquer les données négatives, ou tout résultat inattendu.

La falsification ou la fabrication de données constitue un manquement flagrant à l'éthique de la recherche. Un exemple est l'étude frauduleuse qui fait le lien entre l'autisme et le vaccin (9), qui a causé des torts incalculables en minant la confiance du public envers les vaccins administrés systématiquement aux enfants.

Le plagiat doit être soigneusement évité. Pour incorporer les idées ou les résultats de recherches des autres dans n'importe quel article que vous rédigez, il faut citer les références appropriées. Les éditeurs de revues contrôlent les articles au moyen d'un logiciel anti-plagiat avant de déterminer le bien-fondé d'un article pour l'évaluation par les pairs. Des logiciels gratuits sont à la disposition des auteurs qui souhaitent contrôler la reproduction involontaire de contenu, notamment CopyScape, DupliChecker, Plagiarisma, Plagium, Search Engine Reports, SEOTools, Site Liner et Unplag.

La reproduction est la publication d'un article qui est le même ou présente un contenu très proche de celui d'un autre article de l'auteur ou de l'éditeur (8). Le contenu est considéré comme redondant et peut entraîner une double comptabilisation des données. Il faut faire la distinction entre une reproduction et une copublication : en effet, on parle de copublication lorsque le même article est publié dans plus d'une revue sur la même période, approximativement, afin d'en augmenter la portée au sein de différentes disciplines (8). La copublication répond à des critères précis et est réalisée en toute transparence.

Le chevauchement de publications est une variante de la reproduction. II implique habituellement des essais multicentriques et se caractérise par des publications issues de centres uniques, de plusieurs centres, ainsi que de tous les centres. On considère qu'il est contraire à l'éthique, car il peut entraîner une double comptabilisation et fausser la perception du poids de la preuve (10). Il peut être approprié que plus d'une publication provienne d'un essai multicentrique, mais généralement c'est pour tenir compte des résultats secondaires. Les publications secondaires doivent citer l'analyse primaire et toutes les publications d'essais doivent indiquer le numéro d'enregistrement de l'essai (8).

\section{Donner la reconnaissance pertinente}

Il est important de reconnaître le travail de toutes les personnes qui ont contribué à une publication scientifique. La paternité pertinente est capitale pour une publication éthique. Le fait de définir le rôle de chaque auteur constitue une pratique exemplaire. Selon I'International Committee of Medical Journal Editors (ICMJE), les mérites de la paternité doivent être fondés sur les quatre critères suivants : contributions importantes aux travaux de conception ou de création ou à I'acquisition, I'analyse ou l'interprétation des données pour le travail; rédaction de l'article initial ou révision critique du contenu intellectuel important; approbation finale de la version à publier; responsabilité par rapport à l'ensemble des aspects du travail, dans le sens où l'on veille à ce que les questions liées à l'exactitude ou à l'intégrité de n'importe quelle partie du travail fassent l'objet d'une enquête et soient résolues de manière appropriée (11).

Il convient de souligner que la collecte de données ou l'élaboration de logiciels pour une étude ne sont pas des critères de la paternité, et qu'elles n'assurent pas non plus le financement de la recherche; cependant, ce sont des contributions importantes qui doivent être reconnues - soit dans la section Remerciements, soit dans la section Contributions (s'il y en a une). Le mieux est de s'assurer que toutes les personnes mentionnées dans une section Remerciements ou Contributions sont au courant qu'elles ont été identifiées et qu'elles acceptent d'être identifiées. Souvent, les entrepreneurs payés pour réaliser des parties d'une étude ( $p$. ex. les essais en laboratoire, l'élaboration de logiciels, ou la rédaction de l'article) ne sont pas des auteurs, par définition, mais ils méritent tout de même de figurer dans la section Remerciements ou Contributions.

Certaines pratiques contraires à l'éthique dans la paternité comprennent la rédaction invitée et la rédaction anonyme. La rédaction invitée est l'inclusion d'une personne en tant qu'auteur qui ne satisfait pas aux critères de l'ICMJE, et la rédaction anonyme exclut une personne en tant qu'auteur qui satisfait aux critères de I'ICMJE. Fondamentalement, la reconnaissance éthique est une question de transparence. 
II peut y avoir beaucoup de débats au sujet du séquençage des auteurs. L'ordre des auteurs diffère selon la discipline (12). Dans le domaine des sciences de la santé, c'est le premier auteur qui a le plus de poids; l'auteur final a également du poids, comme il s'agit souvent du chercheur principal ou plus expérimenté. Par contre, en économie, les auteurs sont habituellement cités par ordre alphabétique, ce qui suppose une contribution égale aux travaux de recherche. Il est utile de discuter de la paternité dès le début du processus de planification de l'article, puis à nouveau vers la fin de la rédaction de l'article. Cette discussion doit comprendre une évaluation de la paternité par rapport aux critères de l'ICMJE et la prise en compte de la séquence de paternité, qui pourraient changer au fil du temps s'il venait à y avoir des changements dans le niveau de contribution par rapport à ce qui était prévu à l'origine.

\section{Positionnement de votre article}

Une fois que vos recherches sont terminées, vous devez repérer des revues appropriées aux fins de publication. Tous les articles ne peuvent pas ou ne devraient pas être publiés dans une revue prestigieuse ayant le plus grand impact. Les gens peuvent consacrer beaucoup de temps et d'efforts à l'envoi d'articles à des revues qui vont rapidement renvoyer une lettre de refus polie, ou qui vont garder les articles pendant plusieurs mois avant de les refuser, selon l'évaluation par les pairs. Alors, comment choisir la revue à laquelle soumettre un article? Discutez-en avec vos cochercheurs ou vos pairs : qui est le public cible? Qui voudra connaître cette recherche? Quelle est la meilleure revue pour atteindre ce public? Quelles sont les exigences propres à ces revues concernant les soumissions d'articles?

\section{Définition de votre public cible}

Avant de documenter les résultats de son étude, il faut que vous réfléchissiez aux lecteurs potentiels. Est-ce que les résultats de recherche sont les plus appropriés pour un lectorat général ou un groupe de spécialistes? Cela influence le choix de la revue à laquelle vous allez présenter votre article, de même que le style de rédaction que vous adoptez pour la revue.

\section{Choix de trois à cinq revues}

En fonction de votre lectorat cible, vous devez dresser une liste de trois à cinq revues, puis les classer en fonction du facteur d'impact de la revue. Le facteur d'impact est le nombre moyen de citations par article publié dans cette revue, selon le rendement au cours des deux dernières années (13). Soumettez un article à une revue à la fois, en commençant par le début de la liste. En cas de lettre de refus de la part de la revue de votre "plan $A$ », il faut que vous ayez sous la main un "plan $B$ " pour soumettre l'article à une autre revue sur-le-champ. Cela évite d'avoir l'article refusé qui traîne sur votre bureau.

\section{Apprentissage des exigences des revues}

Chaque revue a des instructions à l'intention des auteurs qui sont mentionnées en ligne. Ces instructions décrivent les types d'articles publiés par la revue et fournissent des conseils précis sur le format, sur la longueur des mots, ainsi que sur les éléments à inclure dans une lettre d'accompagnement au moment de la soumission. Vous devez consulter quelques anciens numéros des revues ciblées pour voir des exemples des différents types d'articles qui sont publiés.

\section{Création de la première ébauche}

Maintenant que vous avez défini votre public cible, la revue que vous ciblez en premier lieu ainsi que les exigences connexes, vous êtes prêt à créer la première ébauche. Pour commencer, vous devriez élaborer un résumé de haut niveau qui établit un scénario logique et convaincant qui suit la structure établie pour un article scientifique. Ensuite, avant de commencer à rédiger le texte, vérifiez les guides de rédaction par rapport au type d'étude que vous avez réalisée; cela vous permettra de vous assurer que vous tenez compte des exigences précises en matière de rédaction.

On croit souvent à tort que les publications scientifiques sont simplement des rapports impartiaux au sujet des méthodes et des résultats de recherche. Mais pensez à ceci : il existe plus de 30000 revues biomédicales (14). Nous vivons à une époque de surcharge d'information, les gens deviennent ainsi très sélectifs dans ce qu'ils lisent et se demandent : "Est-ce que c'est important que je lise cela? » La production de rapports objectifs sur les résultats de recherche est nécessaire, mais cela ne suffit pas. Les auteurs efficaces donneront également un contexte approprié et présenteront leur travail de telle sorte que les lecteurs le trouvent intéressant et facile à comprendre. Les sections qui suivent proposent plusieurs façons de présenter au mieux le contexte, les données et les répercussions de votre travail.

\section{Élaboration d'un scénario convaincant}

L'utilisation du terme "scénario » ici ne signifie pas que vous vous efforcez de divertir le lecteur. C'est la façon dont vous "faites valoir vos arguments » à la cour de l'opinion scientifique. II représente la structure de base des articles scientifiques et comprend la raison d'être de l'étude, la question de recherche, la manière dont cette question a été abordée, les résultats trouvés et les raisons pour lesquelles ces constats sont importants (3). Après avoir travaillé pendant des mois (et parfois des années) sur un projet de recherche, il est facile de se perdre dans les détails. L'établissement d'une structure sous-jacente claire et logique pour votre article scientifique dès le départ permet non seulement d'éviter de digresser, mais aussi d'augmenter considérablement sa lisibilité. Le résumé est un excellent endroit pour établir le scénario de votre article. Vous voulez répondre aux questions suivantes: Qu'est-ce que cette recherche? (contexte et objectif); qu'avez-vous fait pour répondre à cette question de recherche? (méthodologie): qu'avez-vous découvert? (résultats); quelles sont les répercussions et les prochaines étapes? (discussion et conclusion). Ensuite, comme l'établissement du thème, chaque section est développée dans I'article. Un résumé bien écrit donne aux lecteurs une "feuille de route $॥ ;$ après lecture, ils sauront de quoi vous allez parler dans l'article.

Une façon de renforcer la logique de votre article est d'utiliser les mêmes termes et le même séquençage de l'information dans chaque section. Par exemple, si l'objectif de votre recherche était l'évaluation de l'acceptabilité et de l'observance d'un 
schéma thérapeutique, vous ne devriez pas décrire la volonté de commencer un traitement dans la section Introduction. Au lieu de cela, notez la façon dont vous avez mesuré l'observance et le respect du traitement dans la section Méthodologie, puis décrivez le nombre de personnes ayant suivi le schéma thérapeutique après avoir accepté de le commencer dans la section Résultats. Si votre objectif de recherche est d'évaluer l'acceptabilité et l'observance du traitement, définissez I'acceptabilité puis le respect dans la section Introduction, déterminez la manière dont vous avez mesuré l'acceptation puis le respect dans la section Méthodologie, puis décrivez vos constatations pour l'acceptation puis le respect dans la section Résultats. Lorsque vous utilisez les mêmes termes dans le même ordre dans les sections Introduction, Méthodologie et Résultats, il est beaucoup plus facile pour le lecteur de saisir rapidement ce que vous avez fait et ce que vous avez découvert.

En outre, il existe plusieurs techniques de rédaction qui vous permettront de rendre votre article plus convaincant et de mobiliser ainsi le lecteur. La première technique consiste à avoir un "sujet accrocheur » ou un point de départ intéressant qui attire le lecteur. Les titres peuvent être accrocheurs; par exemple, un article récent du New England Journal of Medicine s'intitulait : "The Other Victims of the Opioid Epidemic " (15). Ce titre pourrait attirer votre attention, car vous vous demandez immédiatement : "Qui sont les victimes et qui sont les autres victimes? ॥ Un titre convaincant peut poser une question qui motive les gens à lire l'article : « Les scientifiques et les responsables des politiques peuvent-ils travailler ensemble? " (16). L'intérêt des lecteurs est également capté dès la première phrase du résumé, par exemple : "L'émergence et la prévalence des bactéries résistantes aux antibiotiques sont une cause de décès croissante à l'échelle mondiale, ce qui se traduit par un appel mondial à l'action. » (17). C'est une bonne première phrase, car elle donne un sentiment d'urgence et rend le lecteur curieux quant à la nature de l'appel à l'action. Il faut veiller à ne pas verser dans le sensationnel, mais lorsqu'il y a un problème de santé urgent, il est important d'être au courant et de modifier ce que nous faisons, si nécessaire.

\section{Vérification des guides pour la production de rapports}

À titre d'étape finale, avant de commencer à rédiger l'article au complet, vérifiez s'il y a des exigences précises en matière de production de rapports pour le type de recherche que vous avez effectuée; par exemple, si vous avez réalisé une étude expérimentale, vous devrez mentionner l'approbation et le consentement éclairé du Comité d'éthique de la recherche (18). Si vous avez réalisé un examen systématique, incluez un organigramme des études incluses et exclues (19). Certaines revues fournissent aux auteurs des listes de contrôle sur lesquelles figurent les éléments importants à inclure dans différentes sections pour les différents types d'études $(20,21)$. Le réseau Equator (Enhancing the Quality and Transparency of Health Research) rassemble plusieurs lignes directrices relatives à l'établissement de rapports et constitue une ressource utile (22).

\section{Utilisation de l'approche IMRAD}

Lorsque vous commencez à rédiger le texte, utilisez la structure classique d'un article scientifique, à savoir Introduction,
Méthodologie, Résultats et Discussion, à laquelle on fait souvent référence avec l'acronyme IMRAD. Plutôt que d'écrire tout ce qui, à votre connaissance, est lié à votre étude, utilisez chaque section de façon stratégique afin de raconter l'histoire de votre recherche.

Une bonne section Introduction est structurée comme un triangle inversé. Cela signifie que vous commencez par un sujet vaste, puis que vous procédez à un recentrage des lecteurs par étapes logiques jusqu'à ce que vous arriviez à votre question de recherche. Ce recentrage peut être facilité en répondant aux questions suivantes:

- Quel est le problème?

- Pourquoi est-ce important?

- Que savons-nous à ce jour?

- Quelles sont les lacunes au chapitre de nos connaissances?

- Quelle est la question de recherche qui permettra de combler cette lacune?

- Quel était l'objectif de la recherche?

À ce stade, le lecteur voudra savoir ce qui s'est passé et continuera à lire. Le résumé de la documentation est conjugué au présent, car il représente des faits et des principes généralement acceptés. Définissez toutes les abréviations sur la première utilisation, mais n'utilisez que celles qui sont communément acceptées. La présence d'un trop grand nombre d'abréviations diminue la lisibilité. L'introduction est décrite au présent (comme elle rapporte des faits établis).

La section Méthodologie décrit la façon dont l'étude a été menée. II est important d'expliquer la façon dont les méthodes tiennent compte des objectifs de recherche. Il faut fournir assez de détails afin que les autres puissent reproduire votre étude, si nécessaire, pour confirmer que vos résultats sont uniformes et fiables. Il est utile d'avoir des sous-titres. Pour un essai clinique, par exemple, cela pourrait comprendre la population à l'étude, l'intervention, les mesures de résultats, ainsi que l'analyse. Résistez à la tentation de fournir des résultats dans la section Méthodologie. Par exemple, la méthode d'échantillonnage se place dans la section Méthodologie, tandis que le taux de réponse se place dans la section Résultats. La section Méthodologie est décrite au passé (car elle décrit ce que vous avez fait).

La section Résultats décrit ce que l'on a découvert dans l'étude (dans le même ordre d'information établi dans les sections Introduction et Méthodologie). Résistez à la tentation de discuter des résultats ou de les analyser dans la section Résultats. Par exemple, vous pouvez indiquer: "dans cette étude, il y avait plus d'hommes que de femmes "; mais l'étude de ce constat relève de la section Discussion. La section Résultats est décrite au passé (car elle décrit ce que vous avez découvert).

De nombreux lecteurs trouvent que la section Discussion est la partie la plus intéressante de l'article. La première phrase est une occasion de résumer les résultats les plus importants de votre étude. Par exemple, vous pouvez dire : "Les données de surveillance provenant de quatre pays nordiques ont suggéré qu'au moins $25 \%$ des cas d'infection gonococcique étaient liés au voyage » (23). Interprétez vos résultats à la lumière des biais ou des sources d'erreurs possibles. Ensuite, il est important de prendre en considération les points forts et les points faibles de votre étude; comparez-les à d'autres études qui ont des résultats semblables ou différents, tenez compte des répercussions, et 
déterminez les prochaines étapes. La section Discussion est une occasion de livrer vos résultats dans le bloc de connaissances au sens plus large et de tenir compte des éléments requis pour faire progresser davantage la compréhension scientifique. La discussion est décrite au passé, au présent ou au futur selon le contexte.

\section{Création de tableaux et de figures pour mettre en lumière les principaux résultats}

Deux pratiques exemplaires doivent être envisagées au moment de créer des tableaux et des figures. D'abord, pour répondre à la question classique de la médecine factuelle - ces résultats sont-ils applicables à ma population de patients? - vous devez décrire votre population à l'étude (24). Le premier tableau dans une étude clinique, par exemple, compare souvent les caractéristiques démographiques des sujets de recherche et ce que I'on sait sur la population à l'étude. Cela permet aux lecteurs d'évaluer le degré de représentativité de l'échantillon à l'étude. Ensuite, utilisez les tableaux et les figures pour mettre en évidence vos principaux résultats. Ne cédez pas à la tentation de présenter toutes vos données sous forme de tableaux et de figures, ce qui risque d'accabler le lecteur. Vous voulez vous concentrer sur l'objectif de l'étude et répondre à votre question de recherche.

Les tableaux sont utiles pour présenter de grandes quantités de données, et on privilégie les figures pour montrer les tendances au fil du temps. Les titres des tableaux et des figures doivent être " autonomes ", c'est-à-dire qu'ils sont explicites et complets. Pour être complet, inclure la population à l'étude, le type de données présentées et les dates de l'étude. Dans les tableaux, assurez-vous que chaque colonne comprend un en-tête. Assurez-vous que toutes les données sont validées et que tous les sujets de recherche sont représentés (c.-à-d. l'addition des pourcentages doit donner $100 \%$ ). Des ressources supplémentaires sur la préparation des tableaux et des figures sont disponibles $(25,26)$. Reportez-vous au tableau 1 pour obtenir les principaux repères sur ce qu'il faut faire et éviter lors de la rédaction d'articles scientifiques.

Tableau 1 : Principaux repères sur ce qu'il faut faire et éviter lors de la rédaction d'articles scientifiques

\begin{tabular}{|c|c|c|}
\hline Élément & À faire & À éviter \\
\hline Titre & $\begin{array}{l}\text { Utilisez des titres } \\
\text { précis, intéressants } \\
\text { et accrocheurs. } \\
\text { Exemple : "Les } \\
\text { scientifiques et les } \\
\text { responsables des } \\
\text { politiques peuvent-ils } \\
\text { travailler ensemble? " }\end{array}$ & $\begin{array}{l}\text { N'utilisez pas des titres } \\
\text { trop longs comme: } \\
\text { «Une étude modèle } \\
\text { multisectorielle mixte visant } \\
\text { à examiner les facteurs } \\
\text { qui contribuent ou qui } \\
\text { nuisent à la collaboration } \\
\text { entre les scientifiques } \\
\text { et les responsables des } \\
\text { politiques dans des efforts } \\
\text { communs au moyen de } \\
\text { méthodes qualitatives et } \\
\text { quantitatives ». }\end{array}$ \\
\hline Résumé & $\begin{array}{l}\text { Servez-vous du } \\
\text { résumé pour capter } \\
\text { l'attention des } \\
\text { lecteurs et résumer } \\
\text { votre « scénario ». }\end{array}$ & $\begin{array}{l}\text { N'incluez pas de contenu } \\
\text { qui ne figure pas dans } \\
\text { l'article. }\end{array}$ \\
\hline
\end{tabular}

Tableau 1 : Principaux repères sur ce qu'il faut faire et éviter lors de la rédaction d'articles scientifiques (suite)

\begin{tabular}{|c|c|c|}
\hline Élément & À faire & À éviter \\
\hline \multicolumn{3}{|c|}{ Introduction (pourquoi?) } \\
\hline Objectifs & $\begin{array}{l}\text { Énoncez } \\
\text { rigoureusement votre } \\
\text { objectif de manière } \\
\text { à ce que toute la } \\
\text { suite en découle } \\
\text { logiquement. }\end{array}$ & $\begin{array}{l}\text { N'excluez pas l'objectif } \\
\text { ou ne le rattachez pas } \\
\text { simplement de façon } \\
\text { approximative au reste de } \\
\text { l'article. }\end{array}$ \\
\hline \multicolumn{3}{|c|}{ Méthodologie (comment?) } \\
\hline Pertinence & $\begin{array}{l}\text { Veillez à ce que } \\
\text { la méthode } \\
\text { de recherche } \\
\text { corresponde } \\
\text { aux objectifs de } \\
\text { la recherche, et } \\
\text { expliquez comment } \\
\text { elle le fait. Décrivez } \\
\text { les méthodes } \\
\text { suffisamment en } \\
\text { détail pour que } \\
\text { d'autres personnes } \\
\text { puissent répéter } \\
\text { l'étude. }\end{array}$ & $\begin{array}{l}\text { N'utilisez pas l'étude } \\
\text { transversale pour examiner } \\
\text { les associations causales } \\
\text { puisqu'elle ne le permet } \\
\text { pas. N'indiquez pas «notre } \\
\text { étude utilise des méthodes } \\
\text { conventionnelles » sans } \\
\text { inscrire de références. }\end{array}$ \\
\hline \multicolumn{3}{|l|}{ Résultats (quoi?) } \\
\hline Classement & $\begin{array}{l}\text { Ordonnez } \\
\text { la séquence } \\
\text { d'information de } \\
\text { manière à ce que } \\
\text { la section Résultats } \\
\text { fasse suite à l'objectif } \\
\text { d'une façon logique. }\end{array}$ & $\begin{array}{l}\text { Ne présentez pas les } \\
\text { résultats aléatoirement ou } \\
\text { n'incluez pas de résultats } \\
\text { qui ne sont pas pertinents. }\end{array}$ \\
\hline $\begin{array}{l}\text { Autres } \\
\text { renseignements }\end{array}$ & $\begin{array}{l}\text { Incluez seulement } \\
\text { les résultats de votre } \\
\text { étude dans la section } \\
\text { Résultats. }\end{array}$ & $\begin{array}{l}\text { Les résultats des autres } \\
\text { études doivent figurer dans } \\
\text { l'introduction (pour fournir } \\
\text { un contexte) ou dans la } \\
\text { discussion (pour effectuer } \\
\text { une comparaison avec vos } \\
\text { résultats). }\end{array}$ \\
\hline $\begin{array}{l}\text { Utilisation de } \\
\text { tableaux et de } \\
\text { figures }\end{array}$ & $\begin{array}{l}\text { Les tableaux et les } \\
\text { figures doivent mettre } \\
\text { en évidence les } \\
\text { observations clés de } \\
\text { l'étude. Le texte dans } \\
\text { la section Résultats } \\
\text { doit compléter } \\
\text { les tableaux et les } \\
\text { figures, par exemple, } \\
\text { si un tableau } \\
\text { indique "risque } \\
\text { relatif }=8,5 \text {, } \\
P=0,02 \text { " vous } \\
\text { pourriez écrire } \\
\text { dans le texte " une } \\
\text { forte association } \\
\text { statistiquement } \\
\text { significative a été } \\
\text { établie ». }\end{array}$ & $\begin{array}{l}\text { Ne faites pas que répéter } \\
\text { les données des tableaux } \\
\text { et des figures dans le texte } \\
\text { de la section Résultats, par } \\
\text { exemple, si vous écrivez } \\
\text { « le risque relatif était } \\
8,5 \text { et la valeur de } P \text { était } \\
\text { de } 0,02 \text { » vous répétez les } \\
\text { données déjà présentées } \\
\text { dans le tableau et ne } \\
\text { fournissez aucun nouveau } \\
\text { renseignement aux } \\
\text { lecteurs. }\end{array}$ \\
\hline
\end{tabular}


Tableau 1 : Principaux repères sur ce qu'il faut faire et éviter lors de la rédaction d'articles scientifiques (suite)

\begin{tabular}{|c|c|c|}
\hline Élément & À faire & À éviter \\
\hline \multicolumn{3}{|c|}{ Discussion et conclusion (et alors?) } \\
\hline $\begin{array}{l}\text { Principales } \\
\text { conclusions }\end{array}$ & $\begin{array}{l}\text { La première phrase de } \\
\text { la section Discussion } \\
\text { doit traiter votre } \\
\text { objectif de recherche } \\
\text { et mettre en évidence } \\
\text { les observations clés } \\
\text { de votre étude. }\end{array}$ & $\begin{array}{l}\text { Ne faites pas que } \\
\text { résumer les résultats } \\
\text { une deuxième fois sans } \\
\text { interprétation. }\end{array}$ \\
\hline $\begin{array}{l}\text { Résultats } \\
\text { inattendus }\end{array}$ & $\begin{array}{l}\text { Si les résultats } \\
\text { contredisent la } \\
\text { prédiction, cherchez } \\
\text { des sources possibles } \\
\text { de biais, comme la } \\
\text { sélection des sujets, } \\
\text { les méthodes de } \\
\text { collecte de données } \\
\text { et les facteurs de } \\
\text { confusion. }\end{array}$ & $\begin{array}{l}\text { Ne supprimez pas des } \\
\text { résultats seulement parce } \\
\text { qu'ils contredisent la } \\
\text { prédiction. Il pourrait } \\
\text { s'agir des résultats les plus } \\
\text { importants de votre étude. }\end{array}$ \\
\hline $\begin{array}{l}\text { Contribution } \\
\text { aux } \\
\text { connaissances }\end{array}$ & $\begin{array}{l}\text { Décrivez les nouvelles } \\
\text { connaissances que } \\
\text { l'étude a permis } \\
\text { d'acquérir. }\end{array}$ & $\begin{array}{l}\text { Ne dites pas simplement } \\
\text { "notre étude a confirmé } \\
\text { les résultats des études } \\
\text { antérieures ». }\end{array}$ \\
\hline $\begin{array}{l}\text { Forces et } \\
\text { faiblesses }\end{array}$ & $\begin{array}{l}\text { Abordez les forces } \\
\text { et les faiblesses de } \\
\text { l'étude en quelques } \\
\text { paragraphes. }\end{array}$ & $\begin{array}{l}\text { N'exagérez pas les } \\
\text { faiblesses, mais ne les } \\
\text { cachez pas non plus. }\end{array}$ \\
\hline Implications & $\begin{array}{l}\text { Décrivez la façon } \\
\text { dont l'étude peut } \\
\text { guider la pratique } \\
\text { actuelle. Proposez des } \\
\text { orientations futures } \\
\text { pour la recherche. }\end{array}$ & $\begin{array}{l}\text { Ne dites pas simplement } \\
\text { «notre étude a apporté } \\
\text { d'importantes contributions } \\
\text { à la science », ni « cette } \\
\text { étude indique que d'autres } \\
\text { études sont nécessaires». }\end{array}$ \\
\hline
\end{tabular}

\section{Peaufiner l'article}

La plupart des articles sont le fruit d'un travail d'équipe, alors une fois qu'un article a été rédigé, ils doivent être transmis à tous les coauteurs afin d'obtenir leurs commentaires. Utilisez votre propre processus d'évaluation par les pairs à l'interne, puis peaufinez l'article par souci de clarté avant de le soumettre à une revue à comité de lecture. Si votre langue maternelle n'est pas l'anglais, envisagez de faire réviser l'article avant de le soumettre à une revue.

\section{Distribuer l'article aux coauteurs et aux pairs}

Chaque équipe de recherche établit sa propre méthode de rédaction et de révision. Généralement, le premier auteur rédige l'ébauche initiale, puis l'envoie aux autres auteurs afin qu'ils fournissent des commentaires (normalement en utilisant la fonction de suivi des modifications). Le premier auteur intégrera ensuite les commentaires reçus et produira une deuxième ébauche en vue d'une deuxième ronde de commentaires. Ce processus se poursuit jusqu'à ce que tous les auteurs s'accordent sur la structure et la formulation de l'article. Il est également possible que des auteurs rédigent différentes sections de l'article, une fois que les auteurs se sont entendus sur le scénario et la structure. Une difficulté qui se présente couramment lors de la distribution des ébauches d'un article est le contrôle des versions. Vous voudrez peut-être qu'un seul auteur à la fois travaille sur l'ébauche. Si plusieurs auteurs fournissent leurs commentaires simultanément, ils devraient tous les envoyer au premier auteur avant une date limite fixée. Vous pourriez vouloir également mener votre propre processus d'évaluation par les pairs à l'interne. Après avoir été plongé dans un projet pendant des mois ou dans un article pendant des semaines, il est facile de perdre de vue l'essentiel. Une évaluation par les pairs sans insu à l'interne peut aider à solidifier votre article avant qu'il soit soumis à une évaluation par les pairs à l'aveugle à l'externe, laquelle est réalisée par le bureau de rédaction des revues scientifiques.

\section{Appliquer les principes de rédaction claire}

Une bonne rédaction scientifique se démarque par sa précision et sa clarté (5). Selon le classique, The Elements of Style, voici certains trucs qui vous aideront à rédiger de manière claire (27). Vérifiez la première phrase de chaque paragraphe; elle devrait indiquer au lecteur la progression de la logique de votre article et présenter ce que contient le paragraphe. S'il y a lieu, utilisez la voix active. La formulation " nous avons mis au point un protocole » est plus attrayante que la voix passive " un protocole a été mis au point ». Éliminez les mots inutiles tels que "comme il a été mentionné précédemment ». Lorsque c'est possible, utilisez une construction en parallèle ou la répétition d'une forme grammaticale dans une phrase. Par exemple, la phrase "les enfants âgés de 4 à 6 ans doivent recevoir le vaccin $A$; l'administration du vaccin $B$ est conseillée chez les adolescents âgés de 13 à 18 ans " peut être plus claire si l'on utilise une construction en parallèle, comme suit : « Les enfants âgés de 4 à 6 ans devraient recevoir le vaccin $A$; les adolescents de 13 à 18 ans devraient recevoir le vaccin $B$ ». Énoncez des affirmations irréfutables; suscitez l'intérêt du lecteur en relevant les détails qui comptent. De plus, vous ne devez pas rédiger de manière trop complexe. Des ressources sont à votre disposition pour vous aider à décrire les choses en langage clair (28).

\section{Soumettre l'article et être prêt à le réviser}

Lorsque tous les auteurs ont approuvé la version finale, soumettez-la à la revue de votre choix avec une brève lettre explicative indiquant que votre article n'a pas encore été publié et qu'aucun autre journal n'en fait l'étude. II est également utile de préciser pourquoi votre article est pertinent pour les lecteurs de la revue, car cela pourrait influencer la décision du rédacteur en chef de soumettre ou non votre article à une évaluation par les pairs à l'externe.

Une fois que l'article est envoyé, préparez-vous à de nombreuses réponses possibles. Vous pourriez recevoir une lettre de refus polie, ou le rédacteur en chef pourrait formuler des commentaires sur l'article que vous devrez prendre en compte avant qu'il soit soumis à une évaluation par les pairs. Si c'est le cas, il serait bon d'y donner suite rapidement. II se pourrait également que l'article fasse l'objet d'une évaluation par les pairs, puis soit rejeté. Vous devez attentivement tenir compte de tous les commentaires des pairs examinateurs pour deux raisons, même si la revue ne souhaite pas publier votre article. D'abord, il s'agit de conseils gratuits de la part d'éminents 
experts dans le domaine, alors pourquoi ne pas vous en servir pour améliorer votre taux de réussite auprès d'une autre revue? Ensuite, seulement un nombre limité de chercheurs participent au processus d'évaluation par les pairs des revues. Lorsque vous soumettez votre article à une deuxième revue, vous ne voulez pas qu'on vous dise : "J'étais pair examinateur de cet article pour une autre revue, et je vois que les auteurs n'ont pris en considération aucun des commentaires que j'ai formulés ». Si vous décidez de réviser l'article en fonction des commentaires de l'examinateur, n'oubliez pas de passer en revue les directives pour les auteurs pour l'autre revue et de le reformater, au besoin. Enfin, une fois l'évaluation par les pairs terminée, vous pourriez recevoir du rédacteur en chef une lettre d'acceptation conditionnelle accompagnée d'une demande de changements mineurs. Ou encore, vous pourriez recevoir une lettre de rejet, mais dans laquelle on vous invite à soumettre votre article à nouveau, ce qui signifie que vous devez y apporter des révisions considérables. Dans tous les cas, cela indique un intérêt envers votre article lorsqu'il sera révisé.

Les révisions qui sont demandées font généralement l'objet de discussion entre les coauteurs jusqu'à ce qu'ils s'entendent sur la façon de les intégrer. Les révisions peuvent être réparties entre les auteurs ou coordonnées par une seule personne. Habituellement, lorsque les révisions sont entamées, elles ne sont pas aussi angoissantes qu'elles ne le paraissaient au départ, et l'article finit par être plus pertinent et plus clair 'une fois terminé. Une fois les révisions effectuées, procédez à une vérification finale pour vous assurer que le résumé reflète encore le texte qui a été révisé. Encore une fois, les auteurs doivent tous l'approuver avant que l'article révisé soit soumis à la revue.

\section{Discussion}

La recherche doit être publiée pour que la science progresse. Afin d'optimiser les chances de faire publier vos recherches et d'exercer une influence, il est important de faire preuve d'objectivité, et de présenter votre travail d'une façon intéressante et convaincante. Pour ce faire, vous devez être clair, logique et utiliser des techniques oratoires pour susciter l'intérêt du lecteur envers votre recherche. Cela comprend faire en sorte que votre article touche votre public cible, créer un scénario logique et convaincant dans les limites de la structure IMRAD (acronyme anglais signifiant : Introduction, Méthodologie, Résultats et Discussion), établir une approche itérative efficace parmi les coauteurs afin de mettre au point l'article et être prêt à effectuer les révisions de manière à répondre aux exigences de la revue.

La rédaction scientifique efficace est rarement un talent inné. La capacité de rédaction est une aptitude qu'un auteur doit perfectionner au fil de sa carrière. Intéressez-vous à ce qui fait une bonne rédaction. Lorsque vous lisez les travaux d'autres personnes, demandez-vous ce qui rend certains articles plus faciles à lire que d'autres. Envisagez d'être un pair examinateur pour des revues scientifiques afin d'évaluer les articles d'autres auteurs.

\section{Conclusion}

Il est très satisfaisant de publier une recherche convaincante qui influence les gens et qui apporte des contributions à la science.
On y parvient le plus souvent par l'intermédiaire de la synergie de la collaboration avec d'autres intervenants et en ayant un objectif commun qui vise à promouvoir la progression collective de la science.

\section{Conflit d'intérêt}

Aucun.

\section{Déclaration des auteurs}

Les deux auteurs ont travaillé ensemble à la conception et au schéma. $D^{\text {re }}$ Patricia Huston a élaboré la première ébauche, puis les deux auteurs ont participé à plusieurs ébauches pour ensuite approuver la version finale. $D^{\text {re }}$ Patricia Huston est la rédactrice en chef du Relevé des maladies transmissibles au Canada (RMTC) et s'est récusée des décisions éditoriales relatives au présent article. Les décisions ont été prises par la boursière en rédaction médicale, Toju Ogunremi, avec l'aide du membre du comité de rédaction, $\mathrm{D}^{\mathrm{r}}$ Michel Deilgat.

\section{Remerciements}

Nous remercions Andrea Currie et Katie Rutledge-Taylor, qui ont mis sur pied le programme de rédaction pour les épidémiologistes de terrain de l'Agence de la santé publique du Canada. Nous avons eu de nombreuses discussions sur l'art et la science de la rédaction scientifique qui ont guidé ce document, y compris sur le concept de triangle inversé pour la structure d'une introduction efficace.

\section{Références}

1. Gastel B, Day RA. How to Write and Publish a Scientific Paper, 8th edition. Santa Barbara, USA: Greenwood, 2016.

2. Cargill M, O'Connor P. Writing Scientific Research Articles: Strategy and Steps. West Sussex, UK: John Wiley-Blackwell, 2013.

3. Schimel J. Writing Science: How to Write Papers that get Cited and Proposals that get Funded. New York, USA: Oxford University Press USA, 2012.

4. Albert T. Winning the Publications Game: The Smart Way to Write your Paper and get it Published, 4th edition. New York USA, CRC Press Taylor\&Francis Group, 2016.

5. Katz MJ. From Research to Manuscript: A Guide to Scientific Writing. Springer Science \& Business Media; 2009.

6. The Cochrane Collaboration. About us. http://www. cochrane.org/about-us [Consulté le 20 juillet 2017].

7. Hammes GG. The ACS style guide: Effective Communication of Scientific Information. American Chemical Society; 2006. Chapter 1: Ethics in Scientific Publication in: Coghill AM, Garson LR, editors.

8. International Committee of Medical Journal Editors (ICMJE). Recommendations for the conduct, reporting, editing, and publication of scholarly work in medical journals. Mise à jour 2016. http://www.icmje.org/icmje-recommendations.pdf 
9. Godlee F, Smith J, Marcovitch H. Wakefield's article linking MMR vaccine and autism was fraudulent. BMJ. 2011;342:c7452. DOI (http://dx.doi.org/10.1136/

bmj.c7452). PubMed (https://www.ncbi.nlm.nih.gov/ entrez/query.fcgi?cmd=Retrieve\&db=PubMed\&list_ uids $=21209060 \&$ dopt $=$ Abstract).

10. Huston $P$, Moher D. Redundancy, disaggregation, and the integrity of medical research. Lancet. $1996 \mathrm{Apr}$ 13;347(9007):1024-6. DOI (http://dx.doi.org/10.1016/ S0140-6736(96)90153-1). PubMed (https://www.ncbi.nlm. nih.gov/entrez/query.fcgi?cmd=Retrieve \&db=PubMed\&li st_uids $=8606568 \&$ dopt=Abstract).

11. International Committee of Medical Journal Editors (ICMJE), 2017. Defining the role of authors and contributors. http:// www.icmje.org/recommendations/browse/roles-andresponsibilities/defining-the-role-of-authors-and-contributors. html

12. Choi BCK, Pak AWP. Multidisciplinarity, interdisciplinarity and transdisciplinarity in health research, services, education and policy: 2. Promotors, barriers, and strategies of enhancement. Clinical and Investigative Medicine 2007;30:E224-E232. DOI (http://dx.doi.org/10.25011/ cim.v30i6.2950). PubMed (https://www.ncbi.nlm.nih. gov/entrez/query.fcgi?cmd=Retrieve\&db=PubMed\&list_ uids $=18053389 \&$ dopt $=$ Abstract).

13. Garfield E. The history and meaning of the journal impact factor. JAMA. 2006;295(1):90-3. DOl (http://dx.doi. org/10.1001/jama.295.1.90). PubMed (https://www.ncbi.nlm. nih.gov/entrez/query.fcgi?cmd=Retrieve\&db=PubMed\&li st_uids=16391221\&dopt=Abstract).

14. Klassen TP, Jadad AR, Moher D. Guides for reading and interpreting systematic reviews. 1. Getting started. Arch Pediatr Adolesc Med. 1998;152:700-4. DOI (http://dx.doi. org/10.1001/archpedi.152.7.700). PubMed (https://www. ncbi.nlm.nih.gov/entrez/query.fcgi?cmd=Retrieve\&db=PubM ed\&list_uids=9667544\&dopt=Abstract).

15. Glod SA. The Other Victims of the Opioid Epidemic. N Engl J Med. 2017; 376:2101-2102. DOI (http://dx.doi. org/10.1056/NEJMp1702188). PubMed (https://www.ncbi. $\mathrm{nlm}$.nih.gov/entrez/query.fcgi?cmd=Retrieve\&db=PubMed\&l ist_uids=28564563\&dopt=Abstract).

16. Choi BCK, Pang T, Lin V, Puska P, Sherman G, Goddard M, Ackland MJ, Sainsbury P, Stachenko S, Morrison H, Clottey C, Can scientists and policy-makers work together? Journal of Epidemiology and Community Health. 2005;59:632-637. DOI (http://dx.doi.org/10.1136/jech.2004.031765). PubMed (https://www.ncbi.nlm.nih.gov/entrez/query.fcgi?cmd=Retrie $v e \& d b=$ PubMed\&list_uids=16020638\&dopt=Abstract).

17. Ersoy SC, Heithoff DM, Barnes L 5th, Tripp GK, House JK, Marth JD, Smith JW, Mahan MJ. Correcting a Fundamental Flaw in the Paradigm for Antimicrobial Susceptibility Testing. EBioMedicine. 2017 May 29. 20;173-81. DOI (http://dx.doi. org/10.1016/j.ebiom.2017.05.026). PubMed (https://www. ncbi.nlm.nih.gov/entrez/query.fcgi?cmd=Retrieve\&db=PubM ed\&list_uids=28579300\&dopt=Abstract).

18. Groupe consultatif interorganisme en éthique de la recherche. l'Énoncé de politique des trois Conseils : Éthique de la recherche avec des êtres humains. Ottawa Canada:
Conseil de recherches en sciences humaines du Canada, Conseil de recherches en sciences naturelles et en génie du Canada, Instituts de recherche en santé du Canada; 2014. http://www.ger.ethique.gc.ca/fra/policy-politique/initiatives/ tcps2-eptc2/Default/

19. Moher D, LIberati A, Tetzlaff J, Altman DG, The PRISMA Group. Preferred reporting items for systemic reviews and meta-analyses: The PRISMA Statement. PLoS Med. 209;6(7):e1000097. DOl (http://dx.doi.org/10.1371/ journal.pmed.1000097). PubMed (https://www.ncbi.nlm. nih.gov/entrez/query.fcgi?cmd=Retrieve \&db=PubMed\&li st_uids=19621072\&dopt=Abstract)

20. Guide de la déclaration des éclosions. Relevé des maladies transmissibles au Canada. 2015;41(4):81-3. https://www. canada.ca/fr/sante-publique/services/rapports-publications/ releve-maladies-transmissibles-canada-rmtc/numeromensuel/2015-41/rmtc-volume-41-04-2-avril-2015/rmtcvolume-41-04-2-avril-2015-1.html

21. Guide sommaire de présentation des rapports de surveillance. Relevé des maladies transmissibles au Canada. 2015;41(4):84-6. https://www.canada.ca/fr/santepublique/services/rapports-publications/releve-maladiestransmissibles-canada-rmtc/numero-mensuel/2015-41/ rmtc-volume-41-04-2-avril-2015/rmtc-volume-41-04-2avril-2015-2.html

22. The Equator Network (Enhancing the Quality and Transparency of Health Research). Oxford: EQUATOR Network; 2015. http://www.equator-network.org/

23. Beauté J, Cowan S, Hiltunen-Back E, Kløvstad H, Velicko I, Spiteri G. Travel-associated gonorrhoea in four Nordic countries, 2008 to 2013. Euro Surveill. 2017;22(20):pii=30537. DOI (http://dx.doi.org/10.2807/15607917.ES.2017.22.20.30537). PubMed (https://www.ncbi.nlm. nih.gov/entrez/query.fcgi?cmd=Retrieve \&db=PubMed\&li st_uids=28537548\&dopt=Abstract).

24. Centre for Evidence-Based Medicine. Critical Appraisal Tools. Nuffield Department of Primary Care Health Sciences. http:// www.cebm.net/critical-appraisal/ [Consulté le 20 juillet 2017].

25. Lang TA, Secic M. How to Report Statistics in Medicine: Annotated Guidelines for Authors, Editors, and Reviewers. 2nd edition. Philadelphia USA; American College of Physicians Press; 2006.

26. Evergreen Stephanie DH. Presenting Data Effectively: Communicating Your Findings for Maximum Impact. 2nd edition. Thousand Oaks USA; Sage Publications; 2017.

27. Strunk W, White EB. Elements of Style. New York USA: Allyn \& Bacon; 2000.

28. Plain Language Action and Information network (PLAIN). Simple Words and Phrases. http://www. plainlanguage.gov/ howto/wordsuggestions/simplewords.cfm [Consulté en 2017]. 